\title{
Review of: "Classification of regular and chaotic motions in Hamiltonian systems with deep learning"
}

\author{
Shengliang $\mathrm{Pu}^{1}$ \\ 1 East China University of Technology (ECUT)
}

Potential competing interests: The author(s) declared that no potential competing interests exist.

In this manuscript, the authors take an interest in classifying types of motion on a time series data set coming from simple Hamiltonian systems and predicting the character of the dynamics on a model problem of the forced pendulum and an evolution problem in Celestial Mechanics. The presented study on the use of machine learning techniques to characterize chaotic orbits sounds fairly interesting.

Dynamic models regarding the forced pendulum and the spin-orbit problems have been well introduced in the context, then it extends the regular and chaotic behavior of the dynamic models. That is to solve a problem, how to automatically identify the chaotic, librational, or rotational motions from a given time series data set. Or say, to classify time series data. As a consequence, the comparison experiments have been made among indexing methods based on chaos indicators, and among deep learning architectures.

For a specific classification task from different research fields, the concept "accuracy" may present several possible meanings. That is, correctly identifying issues may involve proper or more metrics for evaluating classification outcomes, and making time-series classification more accurate. Therefore, if possible, further clarification regarding this concern would be better. 